\title{
Propositional modal logic with implicit modal quantification
}

\author{
Anantha Padmanabha ${ }^{1[0000-0002-4265-5772]}$ and R Ramanujam ${ }^{1}$ \\ Institute of Mathematical Sciences, HBNI, Chennai, India \\ $\{$ ananthap, jam\}@imsc.res.in
}

\begin{abstract}
Propositional term modal logic is interpreted over Kripke structures with unboundedly many accessibility relations and hence the syntax admits variables indexing modalities and quantification over them. This logic is undecidable, and we consider a variable-free propositional bi-modal logic with implicit quantification. Thus $[\forall] \alpha$ asserts necessity over all accessibility relations and $[\exists] \alpha$ is classical necessity over some accessibility relation. The logic is associated with a natural bisimulation relation over models and we show that the logic is exactly the bisimulation invariant fragment of a two sorted first order logic. The logic is easily seen to be decidable and admits a complete axiomatization of valid formulas. Moreover the decision procedure extends naturally to the 'bundled fragment' of full term modal logic.
\end{abstract}

Keywords: Term modal logic $\cdot$ Implicitly quantified modal logic $\cdot$ Bisimulation invariance Bundled fragment

\section{Introduction}

Propositional multi-modal logics [414] are used extensively in the context of multi-agent systems, or to reason about labelled transition systems. In the former case, $\square_{i} \alpha$ might refer to knowledge or belief of agent $i$ that $\alpha$ holds. In the latter case, $\diamond_{a} \alpha$ may assert the existence of an $a$-labelled transition from the current state to one in which $\alpha$ holds. Such applications include epistemic reasoning [7]6, games [13, system verification [5] and more.

In either of the settings, the indices of modalities come from a fixed finite set. However, the applications themselves admit systems of unboundedly many agents, or infinite alphabets of actions. The former is the case in dynamic networks of processes, and the latter in the case of systems handling unbounded data. In fact, the set of agents relevant for consideration may itself be dynamic, changing with state.

Such motivations naturally lead to modal logics with unboundedly many modalities, and indeed quantification over modal indices. Grove and Halpern 1211] discuss epistemic logics where the agent set is not fixed and the agent names are not common knowledge. Khan et al. 15] use unboundedly many modalities and allow quantification over them to model information systems 
in approximation spaces. Other works on indexed modalities include Passy and Tinchev [20], Gargov and Goranko [10], Blackburn [3].

Term Modal logic(TML), introduced by Fitting, Voronkov and Thalmann 9 offers a natural solution to these requirements. It extends first order logic with modalities of the form $\square_{x} \alpha$ where $x$ is a variable (and hence can be quantified over). Thus we can write a formula of the form: $\forall x \square_{x}\left(p(x) \supset \exists y \diamond_{y} q(x, y)\right)$. Kooi (16]) considers the expressivity of TML in epistemic setting. Wang and Seligman ([23]) introduce a restricted version of TML where we have assignments in place of quantifiers (formulas of the form $[x:=b] K_{x}(\alpha)$ where $b$ is a constant, whose interpretation as an agent will be assigned to $x$ ).

Note that TML extends first order logic, and hence its satisfiability problem is undecidable. In [18] we prove that the problem is undecidable even when the atoms are restricted to boolean propositions (PTML). Hence the question of finding decidable fragments of PTML is well motivated. In [18] we prove that the monodic fragment of PTML is decidable. The monodic fragment is a restriction allowing at most one free variable within the scope of a modality. i.e, every subformula of the form $\square_{x} \alpha$ has $F V(\alpha) \subseteq\{x\}$.

Orlandelli and Corsi 17] consider two decidable fragments: (1) When quantifier occurrence is restricted to the form: $\exists x \square_{x} \alpha$ (denoted by [ $\left.\exists\right] \alpha$ ); (2) Quantifiers appear in a restricted guarded form: $\forall x\left(P(x) \Rightarrow \square_{x} \alpha\right)$ and $\exists x\left(P(x) \wedge \square_{x} \alpha\right)$ (and their duals). The corresponding first order modal logic counter parts of the first of these fragments is studied by Wang [22]. Shtakser (21]) considers a monadic second order version of the restricted guards (with propositional atoms) of the form $\forall X\left(P(X) \Rightarrow \square_{X} \alpha\right)$ and $\exists X\left(P(X) \wedge \square_{X} \alpha\right)$ where $X$ is quantified over subsets of indices and $P$ is interpreted appropriately. These fragments are semantically motivated from their interest in the epistemic logic to model the notions like 'everyone knows' and 'someone knows' and community knowledge (ex: All eye-witnesses know who killed Mary).

Note that when modalities and quantifiers are 'bundled' together and atomic formulas are propositional, $\exists x \square_{x} \alpha$ can be replaced by a variable free modality $[\exists] \alpha$, and similarly $\forall x \square_{x} \alpha$ by $[\forall]$. In some sense this is the most natural variable free fragment of PTML with modalities being implicitly quantified. This is the logic IQML studied in this paper.

Just as propositional modal logic is the bisimulation-invariant fragment of first order logic, we show that IQML is the bisimulation-invariant fragment of an appropriate two-sorted first order logic. The notion of bisimulation needs to be carefully re-defined to account for quantification over edge labels. Other natural questions on IQML such as decidability of satisfiability and complete axiomatization of valid formulas are answered easily. Interestingly, the natural tableau procedure for the logic can be extended to the 'bundled fragment' of TML with predicates of arbitrary arity, by an argument similar to the one developed in [19] (for a 'bundled fragment' of first order modal logic). 


\section{The logic}

We start with PTML, the propositional fragment of Term-Modal logic. Since we will only study its variable free fragment later, we consider here only the pure vocabulary (no constant and function symbols) with only variables as terms and without equality.

Definition 1 (PTML syntax). Let $\mathcal{V}$ be a countable set of variables and $\mathcal{P}$ be a countable set of propositions. The syntax of PTML is given by:

$$
\varphi:=p|\neg \varphi| \varphi \wedge \varphi|\exists x \varphi| \diamond_{x} \varphi
$$

where $p \in \mathcal{P}$ and $x \in \mathcal{V}$.

The boolean operators $\vee, \supset$ are defined in the standard way. The dual operators for quantifiers and modalities are given by $\forall x \varphi=\neg \exists x \neg \varphi$ and $\square_{x} \varphi=$ $\neg \vee_{x} \neg \varphi$. The notion of free variables $\mathrm{Fv}(\varphi)$ and modal depth $\mathrm{md}(\varphi)$ are standard.

In the semantics, unlike classical modal logics, the agent set is not fixed, but specified along with the structure. Thus the Kripke frame for PTML is given by $(\mathcal{W}, \mathcal{D}, \mathcal{R})$ where $\mathcal{W}$ is a set of worlds, $\mathcal{D}$ is a potential set of agents and $\mathcal{R} \subseteq(\mathcal{W} \times \mathcal{D} \times \mathcal{W})$. The agent dynamics is captured by a function $\left(\delta: \mathcal{W} \rightarrow 2^{\mathcal{D}}\right.$ below) that specifies, at any world $w$, the set of agents alive (or meaningful) at $w$. Then coherence demands that whenever $(u, d, v) \in \mathcal{R}$, we have that $d \in \delta(u)$ : only an agent alive at $u$ can consider $v$ accessible.

A monotonicity condition is imposed on the accessibility relation as well: whenever $(u, d, v) \in \mathcal{R}$, we have that $\delta(u) \subseteq \delta(v)$. This is required to handle interpretations of free variables. Hence the models are called 'increasing agents' models. For more details on this restriction, refer [89].

Definition 2 (PTML structure). An (increasing agent) model for PTML is a tuple $\mathcal{M}=(\mathcal{W}, \mathcal{D}, \mathcal{R}, \delta, \rho)$ where, $\mathcal{W}$ is a non-empty set of worlds, $\mathcal{D}$ is a nonempty set of agents, $\mathcal{R} \subseteq(\mathcal{W} \times \mathcal{D} \times \mathcal{W}), \delta: \mathcal{W} \rightarrow 2^{\mathcal{D}}$ assigns to each $w \in \mathcal{W}$ a non-empty local agent set s.t. $(w, d, v) \in \mathcal{R}$ implies $d \in \delta(w) \subseteq \delta(v)$ for any $w, v \in \mathcal{W}$, and $\rho: \mathcal{W} \mapsto 2^{\mathcal{P}}$.

To interpret free variables, we need a variable assignment function (interpretation) $\sigma: \mathcal{V} \mapsto \mathcal{D}$. Call $\sigma$ relevant at $w \in \mathcal{W}$ for a formula $\varphi$ if $\sigma(x) \in \delta(w)$ for all $x \in \operatorname{Fv}(\varphi)$. The increasing agent condition ensures that whenever $\sigma$ is relevant at $w$ for $\varphi$ and we have $(w, d, v) \in \mathcal{R}$, then $\sigma$ is relevant at $v$ for all subformulas of $\varphi$.

Definition 3 (PTML semantics). Given a model $\mathcal{M}$, a formula $\varphi, w \in \mathcal{W}^{\mathcal{M}}$, and an interpretation $\sigma$ that is relevant at $w$ for $\varphi$, define $\mathcal{M}, w, \sigma \models \varphi$ inductively as follows:

$$
\begin{aligned}
& \mathcal{M}, w, \sigma \models p \Leftrightarrow p \in \rho(w) \\
& \mathcal{M}, w, \sigma \models \neg \varphi \Leftrightarrow \mathcal{M}, w, \sigma \models \varphi \\
& \mathcal{M}, w, \sigma \models(\varphi \wedge \psi) \Leftrightarrow \mathcal{M}, w, \sigma \models \varphi \text { and } \mathcal{M}, w, \sigma \models \psi \\
& M, w, \sigma \models \exists x \varphi \Leftrightarrow \text { there is some } d \in \delta(w) \text { such that } \mathcal{M}, w, \sigma[x \mapsto d] \models \varphi \\
& \mathcal{M}, w, \sigma \models \nabla_{x} \varphi \Leftrightarrow \text { there is some } v \in W^{\mathcal{M}} \text { such that }(w, \sigma(x), v) \in \mathcal{R}^{\mathcal{M}} \\
& \text { and } \mathcal{M}, v, \sigma \models \varphi
\end{aligned}
$$


where $\sigma[x \mapsto d]$ denotes an interpretation that is the same as $\sigma$ except for mapping $x$ to $d$.

Note that $\mathcal{M}, w, \sigma \models \varphi$ is inductively defined only when $\sigma$ is relevant at $w$. A formula $\varphi$ is satisfiable, if there is some $\mathcal{M}$ and some $w \in \mathcal{W}^{\mathcal{M}}$ and an interpretation $\sigma$ which is relevant at $w$ for $\varphi$ such that $\mathcal{M}, w, \sigma \models \varphi$. Also, $\varphi$ is valid if $\neg \varphi$ is not satisfiable. In [18, we prove that the satisfiability problem for PTML is undecidable.

As discussed in the previous section, we consider the variable free fragment of PTML, with implicit modal quantification (IQML).

Definition 4 (IQML syntax). Let $\mathcal{P}$ be a countable set of propositions. The syntax of IQML is given by:

$$
\varphi:=p \in \mathcal{P}|\neg \varphi| \varphi \wedge \varphi|[\exists] \varphi|[\forall] \varphi
$$

Note that, $[\exists] \varphi$ translates to $\exists x \square_{x} \varphi$ in PTML. Similarly $[\forall] \varphi$ translates to $\forall x \square_{x} \varphi$. Since there are no variables in IQML, it is closer to classical propositional modal logics where the set of modal indices is not fixed a priori.

The boolean operators $\vee$ and $\supset$ are defined in the standard way. Also we define $\langle\forall\rangle \varphi=\neg[\exists] \neg \varphi$ and $\langle\exists\rangle \varphi=\neg[\forall] \neg \varphi$ to be the respective duals of the modal operators.

In classical modal logics, the Kripke structure for $n$ modalities is given by $\mathcal{M}=\left(\mathcal{W}, \mathcal{R}_{1}, \cdots \mathcal{R}_{n}, \rho\right)$ where each $R_{i} \subseteq(\mathcal{W} \times \mathcal{W})$ is the accessibility relation for the corresponding index and $\rho$ is the valuation of propositions at every world. But in case of IQML, the modal index set is specified along with the model.

Definition 5 (IQML structure). An IQML structure is given by $\mathcal{M}=\left(\mathcal{W}, \mathbb{R}_{\mathbb{I}}, \rho\right)$ where $\mathcal{W}$ is a non-empty set of worlds, $\mathbb{I}$ is a non-empty countable index set and $\mathbb{R}=\left\{\mathcal{R}_{i} \mid i \in \mathbb{I}\right\}$ where each $\mathcal{R}_{i} \subseteq(\mathcal{W} \times \mathcal{W})$ and $\rho: \mathcal{W} \mapsto 2^{\mathcal{P}}$ is the valuation function.

Note that II could be finite or countably infinite. Hence we assume II to be some initial segment of $\mathbb{N}$ or $\mathbb{N}$ itself. Thus we often denote the model as $\mathcal{M}=$ $\left(\mathcal{W},\left[\mathcal{R}_{1}, \mathcal{R}_{2} \cdots\right], \rho\right)$ when $\mathbb{I}$ is clear from the context. Given a model $\mathcal{M}$, we refer to $\mathcal{W}^{\mathcal{M}}$ etc. to denote its corresponding components. The semantics is defined naturally as follows:

Definition 6 (IQML semantics). Given a model $\mathcal{M}$, a formula $\varphi, w \in \mathcal{W}^{\mathcal{M}}$, define $\mathcal{M}, w \models \varphi$ inductively as follows:

$$
\begin{array}{|ll|}
\mathcal{M}, w \models p & \Leftrightarrow p \in \rho(w) \\
\mathcal{M}, w \models \neg \varphi & \Leftrightarrow \mathcal{M}, w, \forall \varphi \\
\mathcal{M}, w \models(\varphi \wedge \psi) \Leftrightarrow \mathcal{M}, w \models \varphi \text { and } \mathcal{M}, w \models \psi \\
M, w \models[\exists] \varphi & \Leftrightarrow \text { there is some } i \in \mathbb{I} \text { such that for all } u \in \mathcal{W} \\
& \text { if }(w, u) \in R_{i} \text { then } \mathcal{M}, u \models \varphi \\
\mathcal{M}, w \models[\forall] \varphi & \Leftrightarrow \text { for all } i \in \mathbb{I} \text { and for all } u \in \mathcal{W} \\
& \text { if }(w, u) \in R_{i} \text { then } \mathcal{M}, u \models \varphi
\end{array}
$$


The formula $\varphi \in$ IQML is satisfiable if there is some model $\mathcal{M}$ and $w \in \mathcal{W}$ such that $\mathcal{M}, w \models \varphi$. A formula $\varphi$ is said to be valid if $\neg \varphi$ is not satisfiable.

In the sequel we adopt the following convention. Given any model $\mathcal{M}, w \in \mathcal{W}$ and a formula of the form $[\exists] \varphi$, if $\mathcal{M}, w \models[\exists] \varphi$ and $i \in \mathbb{I}$ is the corresponding witness then we write $\mathcal{M}, w \models \square_{i} \varphi$ (similarly we have $\mathcal{M}, w \models \diamond_{i} \varphi$ for $\langle\exists\rangle \varphi$ ).

\section{Axiom system and completeness}

Table 3 gives a complete axiom system for the valid formulas of IQML.

The axioms and inference rules are standard. Axiom $A 2$ describes the interaction between $[\forall]$ and $\langle\forall\rangle$ operators. The $([\exists] \mathrm{Nec})$ rule is sound since $\mathbb{I}$ is non-empty. Note that the axiom system is similar to the one in [12], except for $([\forall] \mathrm{Nec})$ and $([\exists] \mathrm{Nec})$. This is because IQML has no names, as opposed to the logic considered in [12].

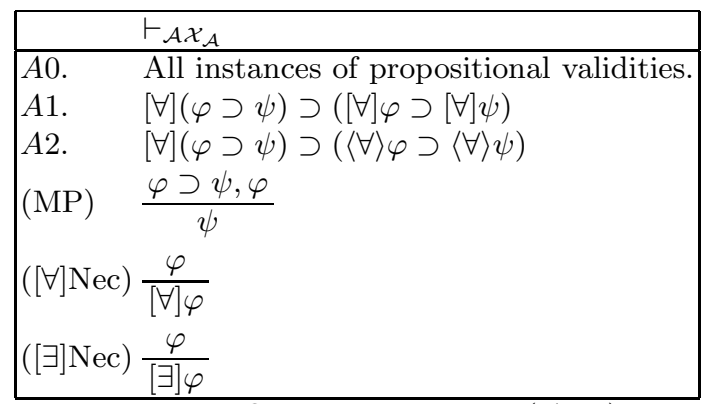

Table 1. IQML axiom system $\left(\mathcal{A} \mathcal{X}_{\mathcal{A}}\right)$

Theorem 1. $\vdash_{\mathcal{A X}}$ is sound and complete for IQML.

We shall first prove soundness.

Lemma 1. The axiom system $\vdash_{\mathcal{A} \mathcal{X}_{\mathcal{A}}}$ is sound for IQML.

Proof. To see that $A 2$ is a validity, for any model $\mathcal{M}$ and any world $w$ let $\mathcal{M}, w \models[\forall](\varphi \supset \psi)$ and $\mathcal{M}, w \models\langle\forall\rangle \varphi$. Since $\mathcal{M}, w \models[\forall](\varphi \supset \psi)$ for any $i \in \mathbb{I}$ and for any $w \stackrel{i}{\rightarrow} u$ we have $\mathcal{M}, u \models \varphi \supset \psi$. Further since $\mathcal{M}, w \models\langle\forall\rangle \varphi$, for any $i \in \mathbb{I}$ there is some $v$ such that $w \stackrel{i}{\rightarrow} v$ and $\mathcal{M}, v \models \varphi$. But then $\mathcal{M}, v \models \varphi \supset \psi$ and hence $\mathcal{M}, v \models \psi$. Thus by semantics, $\mathcal{M}, w \models\langle\forall\rangle \psi$.

Similarly validity of $A 1$ which is the variant of standard $K$ axiom can be verified. Also notice that the inference rules $M P$ and both $(N e c)$ preserve validities. Hence $\vdash_{\mathcal{A} \mathcal{X}_{\mathcal{A}}}$ is sound.

For completeness, we first prove some useful lemmas. The notions of consistent set of formulas and maximally consistent set of formulas is defined in the standard way. 
Lemma 2. For any set of formulas $\Gamma$, if $\Gamma$ is a maximal consistent set then

1. if $\langle\exists\rangle \beta \in \Gamma$ then $\{\beta\} \cup\{\psi \mid[\forall] \psi \in \Gamma\}$ is consistent.

2. if $\{\langle\forall\rangle \gamma,[\exists] \delta\} \subseteq \Gamma$ then $\{\gamma, \delta\} \cup\{\psi \mid[\forall] \psi \in \Gamma\}$ is consistent.

Proof. To prove (1), let $\Gamma$ be a maximal consistent set of formulas and $\langle\exists\rangle \beta \in \Gamma$. Define $\Lambda=\{\beta\} \cup\{\psi \mid[\forall] \psi \in \Gamma\}$. We need to prove that $\Lambda$ is consistent. Suppose not, then there are some $\psi_{1}, \psi_{2} \cdots \psi_{n} \in \Lambda$ such that

$$
\vdash_{\mathcal{A} \mathcal{X}_{\mathcal{A}}}\left(\psi_{1} \wedge \psi_{2} \cdots \psi_{n}\right) \supset \neg \beta
$$

By $([\forall]$ Nec $)$ we have $\vdash_{\mathcal{A} \mathcal{X}_{\mathcal{A}}}[\forall]\left(\left(\psi_{1} \wedge \psi_{2} \cdots \psi_{n}\right) \supset \neg \beta\right)$.

By $(A 1)$ and $(\mathrm{MP}), \vdash_{\mathcal{A} \mathcal{X}_{\mathcal{A}}}[\forall]\left(\psi_{1} \wedge \psi_{2} \cdots \psi_{n}\right) \supset[\forall] \neg \beta$.

Also note that $\left([\forall] \psi_{1} \wedge[\forall] \psi_{2} \cdots[\forall] \psi_{n}\right) \supset[\forall]\left(\psi_{1} \wedge \psi_{2} \cdots \psi_{n}\right)$ is a theorem in this system. Hence $\vdash_{\mathcal{A} \mathcal{X}_{\mathcal{A}}}\left([\forall] \psi_{1} \wedge[\forall] \psi_{2} \cdots \supset \psi_{n}\right) \supset[\forall] \neg \beta$. This implies $[\forall] \neg \beta \in \Gamma$ which is a contradiction to $\langle\exists\rangle \beta \in \Gamma$ and $\Gamma$ is maximally consistent.

To prove (2), again let $\Gamma$ be a maximal consistent set of formulas and let $\{\langle\forall\rangle \gamma,[\exists] \delta\} \subseteq \Gamma$. Define $\Lambda=\{\gamma, \delta\} \cup\{\psi \mid[\forall] \psi \in \Gamma\}$. We need to prove that $\Lambda$ is consistent. Suppose not, then there are some $\psi_{1}, \psi_{2} \cdots \psi_{n} \in \Lambda$ such that $\vdash_{\mathcal{A X}_{\mathcal{A}}}\left(\psi_{1} \wedge \psi_{2} \cdots \psi_{n}\right) \Rightarrow(\gamma \Rightarrow \neg \delta)$.

Now arguing in the same way as in (1) we have

$$
\begin{aligned}
& \Gamma \vdash{ }_{\mathcal{A X}}[\forall](\gamma \Rightarrow \neg \delta) \\
& \Gamma \vdash_{\mathcal{A} \mathcal{X}_{\mathcal{A}}}[\forall](\gamma \Rightarrow \neg \delta) \Rightarrow(\langle\forall\rangle \gamma \Rightarrow\langle\forall\rangle \neg \delta)
\end{aligned}
$$

By $(M P) \quad \Gamma \vdash_{\mathcal{A X}_{\mathcal{A}}}\langle\forall\rangle \gamma \Rightarrow\langle\forall\rangle \neg \delta$

Since $\langle\forall\rangle \beta \in \Gamma, \Gamma \vdash_{\mathcal{A X}_{\mathcal{A}}}\langle\forall\rangle \neg \delta$.

This is a contradiction since $[\exists] \delta \in \Gamma$ and $\Gamma$ is consistent.

Now we define the canonical model. Let $E B=\{[\exists] \alpha \mid[\exists] \alpha \in \mathrm{IQML}\}$ be the set of all $[\exists]$ formulas. These formulas will be used as "agents" in the canonical model.

Definition 7. The canonical model for the propositional packed model is given by $\hat{\mathcal{M}}=\left(\hat{\mathcal{W}}, \hat{\mathcal{R}}_{\hat{\mathbb{I}}}, \hat{\rho}\right)$ where

- $\hat{\mathcal{W}}$ is set of all maximal consistent sets

- $\hat{\mathbb{I}}=\left\{i_{\alpha} \mid \alpha \in E B\right\} \cup\{j\}$ where $j$ is distinct form all $i_{\alpha}$.

- To define $\hat{\mathcal{R}}$, for all $w, u \in \hat{\mathcal{W}}$ and for all $i_{[\exists] \alpha} \in \mathbb{I}$ we have $(w, u) \in \mathcal{R}_{i_{[\exists] \alpha}}$ if $[\exists] \alpha \in w$ and $\{\alpha\} \cup\{\psi \mid[\forall] \psi \in w\} \subseteq u$.

For $j \in \hat{\mathbb{I}}$ we have $w \stackrel{j}{\rightarrow} u$ if $\{\psi \mid[\forall] \psi \in w\} \subseteq u$.

$-\hat{\rho}(w)=w \cap \mathcal{P}$.

Lemma 3. In the canonical model, for any $w, u \in \hat{\mathcal{W}}$ and $i \in \hat{\mathbb{I}}$ if $w \stackrel{i}{\rightarrow} u \in \hat{\mathcal{R}}$ then for all $\psi \in u,\langle\exists\rangle \psi \in w$.

Proof. Suppose not, then there is some $w \stackrel{i}{\rightarrow} u$ and some $\psi \in$ IQML such that $\psi \in u$ but $\langle\exists\rangle \psi \notin w$. But $w$ is maximal, so $[\forall] \neg \psi \in w$. Now since $w \stackrel{i}{\rightarrow} u$, by definition of $\hat{\mathcal{R}}, \neg \psi \in u$ which is a contradiction.

Now we are ready to prove that $\vdash_{\mathcal{A X}}$ is complete for valid formulas of IQML. 
Proof. We show this by proving that any consistent formula $\varphi \in I Q M L$ is satisfiable. First note that any consistent set of formulas $\Gamma$ can be extended to a maximal consistent set by the standard Lindenbaum construction.

Hence for any consistent set of formulas $\Gamma$, there is some world $w \in \hat{\mathcal{W}}$ such that $\Gamma \subseteq w$. Now, we prove the truth lemma.

Claim. For any $w \in \hat{\mathcal{W}}, \hat{\mathcal{M}}, w \models \varphi$ iff $\varphi \in w$.

The proof is by induction on the structure of $\varphi$. In the base case we have propositions and the claim follows by definition of $\hat{\rho}$. The $\neg$ and $\wedge$ cases are standard.

For the case $\varphi:=\langle\exists\rangle \beta$, suppose $\hat{\mathcal{M}}, w \models\langle\exists\rangle \beta$ then there is some $a \in \hat{\gamma}(w)$ and some $(w, a, u) \in \hat{\mathcal{R}}$ such that $\hat{\mathcal{M}}, u \models \beta$. By induction hypothesis $\beta \in u$ and by lemma 3, $\langle\exists\rangle \beta \in w$.

For the other direction, suppose $\langle\exists\rangle \beta \in w$ then since $w$ is a consistent set (by lemma 2(1)) we have $\Gamma=\{\beta\} \cup\{\psi \mid[\forall] \psi \in w\}$ is consistent. Thus there is some world $u \supseteq \Gamma$. Now since $\beta \in u$, by induction hypothesis $\hat{M}, u \models \beta$ and also since $\{\psi \mid[\forall] \psi \in w\} \subseteq u$ we have $w^{\stackrel{j}{\rightarrow}} u$ and hence $\hat{M}, w \models\langle\exists\rangle \beta$.

For the case $\varphi:=[\exists] \beta$, To prove $(\Rightarrow)$, we consider the contrapositive. We prove that if $[\exists] \beta \notin w$ then $\hat{M}, w \models\langle\forall\rangle \neg \beta$. Let $[\exists] \beta \notin w$. Since $w$ is maximally consistent $\langle\forall\rangle \neg \beta \in w$.

Consider any $i_{[\exists] \gamma} \in \hat{\mathbb{I}}$. Now by lemma $2(2), \Gamma=\{\neg \beta, \gamma\} \cup\{\psi \mid[\forall] \psi \in w\}$ is consistent. Thus there is some world $v \supseteq \Gamma$ and by construction of the canonical model, $w \stackrel{i_{[\exists] \gamma}}{\longrightarrow} v$. Also since $\neg \beta \in v$ by induction $\hat{M}, v \models \neg \beta$.

For $j \in \gamma(w)$, let $\top$ be any validity. By $([\exists] \mathrm{Nec})$ we have $\vdash_{\mathcal{A X}_{\mathcal{A}}}[\exists] \top$ and hence $[\exists] \top \in w$. Again, by lemma $2(2), \Gamma=\{\neg \beta, \top\} \cup\{\psi \mid[\forall] \psi \in w\}$ is consistent. Hence there is some $v \supseteq \Gamma$. And thus $w \stackrel{j}{\rightarrow} v$ and by induction hypothesis, $\hat{M}, v \models \neg \beta$.

Thus for every $a \in \gamma(w)$ there is some $v$ such that $w \stackrel{a}{\rightarrow} v$ and $\hat{\mathcal{M}}, v \models \neg \beta$. Hence $\hat{\mathcal{M}}, w \models\langle\forall\rangle \neg \beta$.

For the other direction first note that $[\exists] \beta \in E B$. Now suppose $[\exists] \beta \in w$ then by definition of the canonical model we have for any $w \stackrel{i_{[\exists] \beta}}{\longrightarrow} u$ it is always the case that $\beta \in u$. By induction hypothesis, for any $w \stackrel{i_{[\boxminus] \beta}}{\longrightarrow} u$ we have $\hat{\mathcal{M}}, u \models \beta$. Hence $\hat{\mathcal{M}}, w \models[\exists] \beta$.

\section{IQML bisimulation and elementary equivalence}

Modal logics are naturally associated with bisimulations. If two pointed models are bisimilar, the related worlds agree on propositions and satisfy the so-called "back and forth" property (4 $)$. However, when we come to PTML, since the agent set is not fixed, we need to have the notion of 'world bisimilarity' as well as 'agent bisimilarity'. Towards this, in [18, we introduce a notion of bisimulation for propositional term modal logic and show that it preserves PTML formulas. 
Similar definitions of bisimulations for first order modal logics can be found in 222 .

Now we introduce the notion of bisimulation for IQML. Here the idea is that two worlds are bisimilar if they agree on all propositions and every index in one structure has a corresponding index in the other. The following definition of bisimulation formalizes the notion of 'corresponding index'.

Definition 8. Given two IQML models $\mathcal{M}_{1}$ and $\mathcal{M}_{2}$, an IQML-bisimulation on them is a non-empty relation $G \subseteq\left(\mathcal{W}_{1} \times \mathcal{W}_{2}\right)$ such that for all $\left(w_{1}, w_{2}\right) \in G$ the following conditions hold:

Val. $\rho_{1}\left(w_{1}\right)=\rho_{2}\left(w_{2}\right)$.

$[\exists]$ forth. For all $i \in \mathbb{I}_{1}$ there is some $j \in \mathbb{I}_{2}$ such that, for all $u_{2}$ such that $w_{2} \stackrel{j}{\rightarrow} u_{2}$, there is some $u_{1}$ such that $w_{1} \stackrel{i}{\rightarrow} u_{1}$ and $\left(u_{1}, u_{2}\right) \in G$.

$[\exists]$ back. For all $j \in \mathbb{I}_{2}$ there is some $i \in \mathbb{I}_{1}$ such that, for all $u_{1}$ such that $w_{1} \stackrel{i}{\rightarrow} u_{1}$, there is some $u_{2}$ such that $w_{2} \stackrel{j}{\rightarrow} u_{2}$ and $\left(u_{1}, u_{2}\right) \in G$.

$\langle\exists\rangle$ forth. For all $i \in \mathbb{I}_{1}$ and for all $u_{1}$ such that $w_{1} \stackrel{i}{\rightarrow} u_{1}$, there is some $j \in \mathbb{I}_{2}$ and some $u_{2}$ such that $w_{2} \stackrel{j}{\rightarrow} u_{2}$ and $\left(u_{1}, u_{2}\right) \in G$.

$\langle\exists\rangle$ back. For all $j \in \mathbb{I}_{2}$ and for all $u_{2}$ such that $w_{2} \stackrel{j}{\rightarrow} u_{2}$, there is some $i \in \mathbb{I}_{1}$ and some $u_{1}$ such that $w_{1} \stackrel{i}{\rightarrow} u_{1}$ and $\left(u_{1}, u_{2}\right) \in G$.

Given two models $\mathcal{M}_{1}$ and $\mathcal{M}_{2}$ we say that $w_{1}, w_{2}$ are IQML bisimilar if there is some IQML bisimulation $G$ on the models such that $\left(w_{1}, w_{2}\right) \in G$ and denote it $\left(\mathcal{M}_{1}, w_{1}\right) \leftrightarrow\left(\mathcal{M}_{2}, w_{2}\right)$. Also, we say $\left(\mathcal{M}_{1}, w_{1}\right) \equiv_{\text {IQML }}\left(\mathcal{M}_{2}, w_{2}\right)$ if they agree on all IQML formulas i.e, for all $\varphi \in \mathrm{IQML}, \mathcal{M}_{1}, w_{1} \models \varphi$ iff $\mathcal{M}_{2}, w_{2} \models \varphi$.

Theorem 2. For any two models $\mathcal{M}_{1}$ and $\mathcal{M}_{2}$ and any $w_{1} \in \mathcal{W}_{1}$ and $w_{2} \in \mathcal{W}_{2}$, if $\mathcal{M}_{1}, w_{1} \leftrightarrows \mathcal{M}_{2}, w_{2}$ then $\mathcal{M}_{1}, w_{1} \equiv_{\mathrm{IQML}} \mathcal{M}_{2}, w_{2}$.

Proof. Let $\mathcal{M}_{1}, w_{1} \leftrightarrow \mathcal{M}_{2}, w_{2}$ which means there is some IQML bisimulation $G$ such that $\left(w_{1}, w_{2}\right) \in G$. We need to show that for any $\varphi \in$ IQML we have $\mathcal{M}_{1}, w_{1} \models \varphi$ iff $\mathcal{M}_{2}, w_{2} \models \varphi$.

We prove this for all $\left(v_{1}, v_{2}\right) \in G$ by induction on structure of $\varphi$. The base case and boolean cases are routine.

For the case $\varphi:=[\exists] \psi$ : Suppose $\mathcal{M}_{1}, v_{1} \models[\exists] \psi$, we need to prove that $\mathcal{M}_{2}, v_{2} \models[\exists] \psi$. Since $\mathcal{M}_{1}, v_{1} \models[\exists] \psi$, there is some $i \in \mathbb{I}_{1}$ such that $\mathcal{M}_{1}, v_{1} \models$ $\square_{i} \psi$. Now let $j \in \mathbb{I}_{2}$ be the witness for $i$ for condition ([ $]$ ]forth). We claim that $\mathcal{M}_{2}, v_{2} \models \square_{j} \psi$. Suppose not; then $\mathcal{M}_{2}, v_{2} \models \diamond_{j} \neg \psi$ and hence there is some $v_{2} \stackrel{j}{\rightarrow} u_{2}$ such that $\mathcal{M}_{2}, u_{2} \not \models \psi$. Since $j$ was the witness for $i$ for ([ $\left.\exists\right]$ forth) condition, there is some $w_{1} \stackrel{i}{\rightarrow} u_{1}$ such that $\left(u_{1}, u_{2}\right) \in G$. By induction hypothesis, $\mathcal{M}_{1}, u_{1} \not \models \psi$ which contradicts $\mathcal{M}_{1}, u_{1} \models \square_{i} \psi$. The other direction is proved symmetrically using ([ق]back) condition.

For the case $\langle\exists\rangle \psi$ : Suppose $\mathcal{M}_{1}, v_{1} \models\langle\exists\rangle \psi$ then there is some $i \in \mathbb{I}_{1}$ and some $u_{1} \in W_{1}$ such that $v_{1} \stackrel{i}{\rightarrow} u_{1}$ and $\mathcal{M}_{1}, u_{1} \models \psi$. By condition ( $\langle\exists\rangle$ forth) there is 
some $j \in \mathbb{I}_{2}$ and some $v_{2} \stackrel{j}{\rightarrow} u_{2}$ such that $\left(u_{1}, u_{2}\right) \in G$. By induction hypothesis $M_{2}, u_{2} \models \psi$ and hence $\mathcal{M}_{2}, v_{2} \models\langle\exists\rangle \psi$. The other direction is symmetrically argued using $(\langle\exists\rangle$ back $)$ condition.

Now we prove that the converse holds over image finite models with finite index set $(\mathbb{I}) . \mathcal{M}$ is said to be (index, image) finite if $\mathbb{I}$ is finite and $N^{i}(w)=\{u \mid$ $\left.(w, u) \in R_{i}\right\}$ is finite for all $w \in \mathcal{W}$ and $i \in \mathbb{I}$.

Theorem 3. Suppose $\mathcal{M}_{1}$ and $\mathcal{M}_{2}$ are (index,image) finite models then $\mathcal{M}_{1}, w_{1} \leftrightarrows \mathcal{M}_{2}, w_{2}$ iff $\mathcal{M}_{1}, w_{1} \equiv_{\mathrm{IQML}} \mathcal{M}_{2}, w_{2}$.

Proof. $(\Rightarrow)$ follows from Theorem 2 ,

For $(\Leftarrow)$ suppose $\mathcal{M}_{1}, w_{1} \equiv_{\mathrm{IQML}} \mathcal{M}_{2}, w_{2}$, then define $G=\left\{\left(v_{1}, v_{2}\right) \mid \mathcal{M}_{1}, v_{1} \equiv_{\mathrm{IQML}}\right.$ $\left.\mathcal{M}_{2}, v_{2}\right\}$. Note that $\left(w_{1}, w_{2}\right) \in G$. Hence it suffices to show that $G$ is indeed an IQML bisimulation. For this, choose any $\left(v_{1}, v_{2}\right) \in G$. Clearly $[\mathrm{Val}]$ holds since $v_{1}, v_{2}$ agree on all IQML propositions. Now we verify the other conditions:

Now suppose that the ([ $\exists]$ forth) condition does not hold. Then there is some $\mathbf{i} \in \mathbb{I}_{1}$ such that for all $j \in \mathbb{I}_{2}$ there is some $u_{j}(*)$ such that $v_{2} \stackrel{j}{\rightarrow} u_{j}$ and for all $v_{1} \stackrel{\mathrm{i}}{\rightarrow} u^{\prime}$ we have $u^{\prime} \not \mathrm{FIQML}_{\mathrm{I}} u_{j}$. Let $\mathbb{I}_{2}=\left\{j_{1} \cdots j_{n}\right\}$ and let $u_{l}$ be the corresponding $(*)$ for every $j_{l}$. Also let $\mathbf{i}$-successors of $v_{1}$ be $N_{\mathbf{i}}\left(v_{1}\right)=\left\{s_{1} \cdots s_{m}\right\}$. By above argument, we have $u_{l}$ 三 IQML $_{\mathrm{I}_{d}}$ for all $l \leq n$ and $d \leq m$. Hence for every $u_{l}$ and every $s_{d} \in N_{\mathbf{i}}\left(v_{1}\right)$ there is a formula $\varphi_{d}^{l}$ such that $\mathcal{M}_{1}, s_{d} \models \varphi_{d}^{l}$ but $\mathcal{M}_{2}, u_{l} \models \neg \varphi_{d}^{l}$. Now consider the formula $\alpha=[\exists]\left(\bigwedge_{l} \bigvee_{d} \varphi_{d}^{l}\right)$. Note that for all $l$ and for all $\mathbf{i}$-successors $s_{d} \in N_{\mathbf{i}}\left(v_{1}\right)$ we have $\mathcal{M}_{1}, s_{d} \models \varphi_{d}^{l}$ and hence $\mathcal{M}_{1}, v_{1} \models$ $\square_{\mathbf{i}}\left(\bigwedge_{l} \bigvee_{d} \varphi_{d}^{l}\right)$ which implies $\mathcal{M}_{1}, w_{d} \models \alpha$. On the other hand for every $j_{l} \in \mathbb{I}_{2}$ at $u_{l}$ we have $\mathcal{M}_{2}, u_{l} \models \bigwedge_{d} \neg \varphi_{d}^{l}$ and hence $\mathcal{M}_{2}, v_{2} \models\langle\forall\rangle\left(\bigvee_{l} \bigwedge_{d} \neg \varphi_{d}^{l}\right)$ which contradicts $v_{1} \equiv \mathrm{IQML} v_{2}$.

The ([ $]$ back) condition is argued symmetrically.

Suppose that the $(\langle\exists\rangle$ back $)$ condition does not hold. Then there is some $\mathbf{j} \in \mathbb{I}_{2}$ and some $w_{2} \stackrel{j}{\rightarrow} \mathbf{u}_{2}$ such that for all $i \in \mathbb{I}_{1}$ and for all $w_{1} \stackrel{i}{\rightarrow} u^{\prime}$ we have $u^{\prime} \not \mathrm{IQMML}_{\mathrm{IQ}} \mathbf{u}_{2}$. Let $\mathcal{R}=\bigcup_{i \in \mathbb{I}_{1}} \mathcal{R}_{i}$ and let $N\left(w_{1}\right)=\left\{u^{\prime} \mid\left(v_{1}, u^{\prime}\right) \in \mathcal{R}\right\}$ be the set of all successors of $w_{1}$. Since $\mathcal{M}_{1}$ is (index, image) finite, let $N\left(w_{1}\right)=\left\{t_{1} \cdots t_{r}\right\}$. By above argument, for every $t_{d} \in N\left(w_{1}\right)$ there is a formula $\psi_{d}$ such that $\mathcal{M}_{1}, t_{d} \models \psi_{d}$ and $\mathcal{M}_{2}, \mathbf{u}_{2} \models \neg \psi_{d}$. Hence $\mathcal{M}_{2}, w_{2} \models \diamond_{\mathbf{j}}\left(\bigwedge_{d} \neg \psi_{d}\right)$. Now consider $\beta=\langle\exists\rangle\left(\bigwedge_{d} \neg \psi_{d}\right)$. Clearly $\mathcal{M}_{2}, w_{2} \models \beta$ (with $\mathbf{j}$ and $\mathbf{u}_{2}$ as witnesses). On the other hand, for any successor $t_{d}$ of $w_{1}$ since $\mathcal{M}_{1}, t_{d} \models \psi_{d}$ we have $\mathcal{M}_{1}, w_{1} \models[\forall]\left(\bigvee_{d} \psi_{d}\right)$ which contradicts our assumption that $w_{1}$ and $w_{2}$ satisfy the same formulas. The $(\langle\exists\rangle$ forth $)$ is argued symmetrically.

An important consequence of the theorem above is that we can confine ourselves to tree models for IQML formulas, since it is easily seen that an IQML model is bisimilar to its tree unravelling. 
Given a tree model $\mathcal{M}$ we define its restriction to level $n$ in the obvious manner: $\mathcal{M} \mid n$ is simply the same as $\mathcal{M}$ upto level $n$ and the remaining nodes in $\mathcal{M}$ are 'thrown away'.

We can now sharpen the result above: we can define a notion of $n$-bisimilarity and show that it preserves IQML formulas with modal depth at most $n$.

Definition 9. Given two tree models $\mathcal{M}_{1}$ and $\mathcal{M}_{2}$, and $w_{1}$ in $M_{1}$, w2 in $M_{2}$, we say $w_{1}$ and $w_{2}$ are 0 -bisimilar if $\rho_{1}\left(w_{1}\right)=\rho_{2}\left(w_{2}\right)$.

For $n>0$, we say $w_{1}$ and $w_{2}$ are $n$-bisimilar if the following conditions hold:

$n$-[ $\exists]$ forth. For all $i \in \mathbb{I}_{1}$ there is some $j \in \mathbb{I}_{2}$ such that for all $w_{2} \stackrel{j}{\rightarrow} u_{2}$ there is some $w_{1} \stackrel{i}{\rightarrow} u_{1}$ such that $u_{1}$ and $u_{2}$ are $(n-1)$-bisimilar.

$n$-[ヨ]back. For all $j \in \mathbb{I}_{2}$ there is some $i \in \mathbb{I}_{1}$ such that for all $w_{1} \stackrel{i}{\rightarrow} u_{1}$ there is some $w_{2} \stackrel{j}{\rightarrow} u_{2}$ such that $u_{1}$ and $u_{2}$ are $(n-1)$-bisimilar.

$n-\langle\exists\rangle$ forth. For all $i \in \mathbb{I}_{1}$ and for all $w_{1} \stackrel{i}{\rightarrow} u_{1}$ there is some $j \in \mathbb{I}_{2}$ and some $w_{2} \stackrel{j}{\rightarrow} u_{2}$ such that $u_{1}$ and $u_{2}$ are $(n-1)$-bisimilar.

$n-\langle\exists\rangle$ back. For all $j \in \mathbb{I}_{2}$ and for all $w_{2} \stackrel{j}{\rightarrow} u_{2}$ there is some $i \in \mathbb{I}_{1}$ and some $w_{1} \stackrel{i}{\rightarrow} u_{1}$ such that $u_{1}$ and $u_{2}$ are $(n-1)$-bisimilar.

We can now speak of an $n$-bisimulation relation between models and speak of models being $n$-bisimilar, and employ the notation $\left(\mathcal{M}_{1}, w_{1}\right) \unlhd_{n}\left(\mathcal{M}_{2}, w_{2}\right)$. Clearly, for tree models $\left(\mathcal{M}_{1}, w_{1}\right) \uplus_{n}\left(\mathcal{M}_{2}, w_{2}\right)$ iff $\left(M_{1} \mid n, w_{1}\right) \leftrightarrows\left(M_{2} \mid n, w_{2}\right)$.

A routine re-working of the proof of Theorem 2 shows that when two tree models are $n$-bisimilar, they satisfy the same formulas of modal depth at most

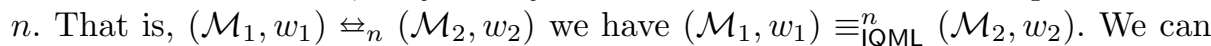
go further and show that every $n$-bisimulation class is represented by a single formula of modal depth at most $n$. For this, we assume (as is customary in modal logic), that we have only finitely many atomic propositions.

Lemma 4. Suppose that $\mathcal{P}$ is a finite set, then for any $n$ and for any $\mathcal{M}, w$ there is a formula $\chi_{[\mathcal{M}, w]}^{n} \in I Q M L$ of modal depth $n$ such that for any $\left(\mathcal{M}^{\prime}, w^{\prime}\right) \models$ $\chi_{[\mathcal{M}, w]}^{n}$ iff $\left(\mathcal{M}^{\prime}, w^{\prime}\right) \leftrightarrows_{n}(\mathcal{M}, w)$.

Proof. Note that $(\Leftarrow)$ follows from Theorem 2 specialized to $n$-bisimulation. For the other direction, the proof is by induction on $n$. For $n=0$, since $\mathcal{P}$ is finite, $\chi_{[\mathcal{M}, w]}^{0}=\bigwedge_{p \in \rho(w)} p \wedge \bigwedge_{q \notin \rho(w)} \neg q$ is the required formula.

Let $\mathcal{R}=\bigcup \mathcal{R}_{i}$ and let $\Gamma_{\mathcal{M}}^{n}=\left\{\chi_{[\mathcal{M}, w]}^{n} \mid w \in \mathcal{W}\right\}$. Inductively $\Gamma_{\mathcal{M}}^{n}$ is finite. For any $S \subseteq \Gamma_{\mathcal{M}}^{n}$ let $\stackrel{\vee}{S}$ denote the disjunction $\bigvee_{\varphi \in S} S$. For the induction step, the 
characteristic formula is given by:

$$
\begin{aligned}
\chi_{[\mathcal{M}, w]}^{n+1}= & \overbrace{\chi_{[\mathcal{M}, w]}^{0}}^{V a l .} \wedge \overbrace{\bigwedge_{i \in \mathbb{I}}[\exists]\left(\bigvee_{(w, u) \in \mathcal{R}_{i}} \chi_{[\mathcal{M}, u]}^{n}\right)}^{n-[\exists] \text { forth }} \wedge \overbrace{\bigwedge_{S \subseteq \Gamma_{\mathcal{M}}^{n}}\left([\exists](S) \supset \bigvee_{i \in \mathbb{I}}^{\vee} \bigwedge_{(w, u) \in \mathcal{R}_{i}}[\forall]\left(\chi_{[\mathcal{M}, u]}^{n} \supset \stackrel{V}{S}\right)\right)}^{n-[\exists] \text { back }} \\
& \underbrace{\bigwedge_{(w, u) \in \mathcal{R}}\langle\exists\rangle \chi_{[M, u]}^{n}}_{n-\langle\exists\rangle \text { forth }} \wedge \underbrace{[\forall]\left(\bigvee_{(w, u) \in \mathcal{R}} \chi_{[M, u]}^{n}\right)}_{n-\langle\exists\rangle \text { back }}
\end{aligned}
$$

Note that the formula remains finite even if $\mathbb{I}$ is infinite or the number of successors of $w$ is infinite since inductively there are only finitely many characteristic formulas of depth $n$. We now prove that the formula $\chi_{\mathcal{M}, w]}^{n}$ indeed captures $n$-bisimulation. First we verify that the formula $\chi_{[\mathcal{M}, w]}^{n}$ holds at $\mathcal{M}, w$ :

- $\mathcal{M}, w \models \chi_{[\mathcal{M}, w]}^{0}$ follows from the definition of $\rho$.

- For the $n-[\exists]$ forth part, for every $i \in \mathbb{I}$ we have $\mathcal{M}, w \models \square_{i}\left(\underset{(w, u) \in \mathcal{R}_{i}}{\bigvee} \chi_{[\mathcal{M}, u]}^{n}\right)$ and hence the claim follows.

- For the $n-[\exists]$ back part, let $S \subseteq \Gamma_{\mathcal{M}}^{n}$. Suppose $\mathcal{M}, w \models[\exists] \stackrel{\vee}{S}$, let $\mathbf{j}$ be the witness. Hence we have $\mathcal{M}, w \models \square_{\mathbf{j}} S$. Now observe that for all $(w, u) \in \mathcal{R}_{\mathbf{j}}$ we have $\chi_{[\mathcal{M}, u]}^{n} \in S$, otherwise there is some $(w, u) \in R_{\mathbf{j}}$ such that $\mathcal{M}, u \models$ $\bigwedge_{\varphi \in S} \neg \varphi$ which is a contradiction to $\mathcal{M}, w \models \square_{\mathbf{j}} \stackrel{\vee}{S}$.

Also, note that for any finite set of formulas $T$, if $\alpha \in T$ then $\alpha \supset\left(\bigvee_{\psi \in T} \psi\right)$ is a propositional validity.

Now we need to show that $\mathcal{M}, w \models \bigvee_{i \in \mathbb{I}} \bigwedge_{(w, u) \in \mathcal{R}_{i}}[\forall]\left(\chi_{[\mathcal{M}, u]}^{n} \supset \stackrel{\vee}{S}\right)$. For this, pick $i=\mathbf{j}$. By above argument, for all $(w, u) \in \mathcal{R}_{\mathbf{j}}$ we have $\left(\chi_{[\mathcal{M}, u]}^{n} \supset \stackrel{\vee}{S}\right)$ as a boolean validity. Hence, we have $\mathcal{M}, w \models \bigwedge_{(w, u) \in \mathcal{R}_{\mathbf{j}}}[\forall]\left(\chi_{[\mathcal{M}, u]}^{n} \supset \stackrel{\vee}{S}\right)$.

- For $n-\langle\exists\rangle$ forth, let $(w, u) \in \mathcal{R}$ which means for some $i \in \mathbb{I}$ we have $(w, u) \in$ $R_{i}$ and such that $\mathcal{M}, u \models \chi_{[\mathcal{M}, u]}^{n}$. Hence $\mathcal{M}, w \models\langle\exists\rangle \chi_{[\mathcal{M}, u]}^{n}$.

- For $n-\langle\exists\rangle$ back, for any $i \in \mathbb{I}$ and any $(w, u) \in R_{i}$ we have $\mathcal{M}, u \models \chi_{[\mathcal{M}, u]}^{n}$ and hence $\mathcal{M}, w \models[\forall]\left(\bigvee_{(w, u) \in \mathcal{R}} \chi_{[\mathcal{M}, u]}^{n}\right)$.

Now suppose $\mathcal{M}^{\prime}, w^{\prime} \models \chi_{[\mathcal{M}, w]}^{n}$, then we need to prove that $\mathcal{M}^{\prime}, w^{\prime}$ is $(n)$-bisimilar to $\mathcal{M}, w$. We verify all the conditions:

- Condition $(\mathrm{Val})$ follows since $\mathcal{M}^{\prime}, w^{\prime} \models \chi_{[\mathcal{M}, w]}^{0}$.

- For condition $\left(n-[\exists]\right.$ forth), let $i \in \mathbb{I}$. By ([ $[\exists]$ forth) part $\chi_{\mathcal{M}, w]}^{n}$ we have $\mathcal{M}^{\prime}, w^{\prime} \models[\exists]\left(\bigvee_{(w, u) \in \mathcal{R}_{i}} \chi_{[\mathcal{M}, u]}^{n}\right)$. Let $\mathbf{j}^{\prime} \in \mathbb{I}^{\prime}$ be the witness such that $\mathcal{M}^{\prime}, w^{\prime} \models$ 
$\square_{\mathbf{j}^{\prime}}\left(\bigvee_{(w, u) \in \mathcal{R}_{i}} \chi_{[\mathcal{M}, u]}^{n}\right)$. Now for any $\left(w^{\prime}, u^{\prime}\right) \in \mathcal{R}_{\mathbf{j}^{\prime}}^{\prime}$ there is some $(w, u) \in R_{i}$ such that $\mathcal{M}^{\prime}, u^{\prime} \models \chi_{[\mathcal{M}, u]}^{n}$ where $(w, u) \in \mathcal{R}_{i}$ and by induction hypothesis, $\left(u, u^{\prime}\right)$ are $n$-bisimilar.

- For condition $(n-[\exists]$ back $)$, let $i^{\prime} \in \mathbb{I}^{\prime}$.

Define $S=\left\{\chi_{[\mathcal{M}, u]}^{n-1} \mid\right.$ for some $\left(w^{\prime}, u^{\prime}\right) \in \mathcal{R}_{i^{\prime}}$ we have $\left.\mathcal{M}^{\prime}, u^{\prime} \models \chi_{[\mathcal{M}, u]}^{n-1}\right\}$. Now clearly, $\left.\mathcal{M}^{\prime}, w^{\prime} \models[\exists] \stackrel{\vee}{S}\right)$. Hence by $(n-[\exists]$ back $)$ part of the formula, there is some $\mathbf{i} \in I$ for which $\mathcal{M}^{\prime}, w^{\prime} \models \bigwedge_{(w, u) \in \mathcal{R}_{i}}[\forall]\left(\chi_{[\mathcal{M}, u]}^{n} \supset \stackrel{\vee}{S}\right)$.

Now, let $T=\left\{\chi_{[\mathcal{M}, u]}^{n-1} \mid\right.$ for some $\left.(w, u) \in \mathcal{R}_{\mathbf{i}}\right\}$. Note that $T \subseteq S$ (otherwise, there is some $(w, u) \in R_{\mathbf{i}}$ such that $\mathcal{M}, u \models \chi_{[\mathcal{M}, u]}^{n-1} \wedge \bigwedge_{\varphi \in S} \neg \varphi$ which implies $\mathcal{M}, w \models \neg \bigwedge_{(w, u) \in \mathcal{R}_{i}}[\forall]\left(\chi_{[\mathcal{M}, u]}^{n} \supset \stackrel{\vee}{S}\right)$ and this is a contradiction to $\mathcal{M}, w \models$ $\left.\chi_{[\mathcal{M}, w]}^{n}\right)$. Hence, for every $(w, u) \in \mathcal{R}_{\mathbf{i}}$ there is some $\left.\left(w^{\prime}, u^{\prime}\right) \in \mathcal{R}\right) i^{\prime}$ such that $\mathcal{M}^{\prime}, u^{\prime} \models \chi_{\mathcal{M}, u]}^{n-1}$. Thus $\mathbf{i}$ is the ([ $]$ ] back) witness for $i^{\prime}$.

- For the $n-\langle\exists\rangle$ forth condition, let $i \in \mathbb{I}$ and $(w, u) \in \mathcal{R}_{i}$. By $n-\langle\exists\rangle$ forth part of the formula, $\mathcal{M}^{\prime}, w^{\prime} \models\langle\exists\rangle \chi_{[\mathcal{M}, u]}^{n-1}$ and hence we have a corresponding $i^{\prime} \in \mathbb{I}^{\prime}$ and $\left(w^{\prime}, u^{\prime}\right) \in \mathcal{R}_{i^{\prime}}$ such that $\mathcal{M}^{\prime}, u^{\prime} \models \chi_{[\mathcal{M}, u]}^{n-1}$.

- Finally for $n-\langle\exists\rangle$ back, suppose $i^{\prime} \in \mathbb{I}$ and $\left(w^{\prime}, u^{\prime}\right) \in \mathcal{R}_{i^{\prime}}$ then by $n-\langle\exists\rangle$ back part of the formula, $\mathcal{M}^{\prime}, u^{\prime} \models \chi_{[\mathcal{M}, u]}^{n-1}$ for some $i \in \mathbb{I}$ and $(w, u) \in R_{i}$. Thus we obtain the required witness.

\section{Bisimulation games and invariance theorem}

Like every propositional modal logic, IQML is also a fragment of first order logic. However, implicit quantification over domain elements in IQML needs to be made explicit as well as quantification over worlds. Since these serve different purposes in the semantics, we use a two sorted first order logic.

Definition 10 (2Sor.FO syntax). Let $\mathcal{V}_{X}$ and $\mathcal{V}_{\tau}$ be two countable and disjoint sorts of variables and $R$ a ternary predicate. The two sorted FO (2Sor.FO), corresponding to IQML is given by:

$$
\alpha::=Q_{p}(x)|R(x, \tau, y)| \neg \alpha|\alpha \wedge \alpha| \exists \tau \alpha \mid \exists x \alpha
$$

where $Q_{p}$ is the corresponding monadic predicate for every $p \in \mathcal{P}$ and $x, y \in \mathcal{V}_{X}$ and $\tau \in \mathcal{V}_{\tau}$.

A 2 Sor.FO structure is given by $\mathfrak{M}=[(\mathcal{W}, \mathbb{I}),(\hat{R}, \hat{\rho})]$ where $(\mathcal{W}, \mathbb{I})$ is the two sorted domain and $(\hat{R}, \hat{\rho})$ are interpretations with $R \subseteq(\mathcal{W} \times \mathbb{I} \times \mathcal{W})$ and $\hat{\rho}: \mathcal{W} \mapsto 2^{Q_{\mathcal{P}}}$ where $Q_{\mathcal{P}}=\left\{Q_{p} \mid p \in \mathcal{P}\right\}$. The semantics $\Vdash$ is defined for 2 Sor.FO in the standard way where the variables in $\mathcal{V}_{X}$ range over the first sort $(\mathcal{W})$ and variables of $\mathcal{V}_{\tau}$ range over second (I). 
Given an IQML structure $\mathcal{M}=\left(\mathcal{W}, \mathcal{R}_{\mathbb{I}}, \rho\right)$ the corresponding 2 Sor.FO structure is given by $\mathfrak{M}=[(\mathcal{W}, \mathbb{I}),(\hat{R}, \hat{\rho})]$ where $(w, i, v) \in \hat{R}$ iff $(w, v) \in \mathcal{R}_{i}$ and $Q_{p} \in \hat{\rho}(w)$ iff $p \in \rho(w)$. Similarly given any 2 Sor.FO structure, it can be interpreted as an IQML structure. Thus there is a natural correspondence between IQML structures and 2Sor.FO structures. For any IQML structure $\mathcal{M}$ let the corresponding 2 Sor.FO structure be denoted by $\mathfrak{M}$.

Definition 11 (IQML to 2 Sor.FO translation). The translation of $\varphi \in I Q M L$ into a 2 Sor.FO parametrized by $x \in \mathcal{V}_{X}$ is given by:

$$
\begin{aligned}
& \operatorname{Tr}(p: x)=Q_{p}(x) \\
& \operatorname{Tr}(\neg \varphi: x)=\neg \operatorname{Tr}(\varphi: x) \\
& \operatorname{Tr}(\varphi \wedge \psi: x)=\operatorname{Tr}(\varphi: x) \wedge \operatorname{Tr}(\psi: x) \\
& \operatorname{Tr}([\exists] \varphi: x)=\exists \tau \forall y(R(x, \tau, y) \supset \operatorname{Tr}(\varphi: y)) \\
& \operatorname{Tr}([\forall] \varphi: x)=\forall \tau \forall y(R(x, \tau, y) \supset \operatorname{Tr}(\varphi: y))
\end{aligned}
$$

Proposition 1. For any formula $\varphi \in \mathrm{IQML}$ and any IQML structure $\mathcal{M}$ $\mathcal{M}, w \models \varphi$ iff $\mathfrak{M},[x \mapsto w] \Vdash \operatorname{Tr}(\varphi: x)$.

Hence IQML can be translated into 2 Sor.FO with 2 variables of $\mathcal{V}_{X}$ sort and one variable of $\mathcal{V}_{\tau}$ sort. Given two IQML models $\mathcal{M}_{1}$ and $\mathcal{M}_{2}$, the notion of IQML bisimulation naturally translates to bisimulation over the corresponding 2Sor.FO models $\mathfrak{M}_{1}$ and $\mathfrak{M}_{2}$.

Now we state the van Benthem type characterization theorem: bisimulation invariant 2Sor.FO formulas can be translated back into IQML. We say that $\alpha(x) \in$ 2Sor.FO is bisimulation invariant if for all $\mathcal{M}_{1}, w_{1} \leftrightarrow \mathcal{M}_{2}, w_{2}$ we have $\mathfrak{M}_{1},[x \mapsto$ $\left.w_{1}\right] \Vdash \alpha(x)$ iff $\mathfrak{M}_{2},\left[x \mapsto w_{2}\right] \Vdash \alpha(x)$. We can similarly speak of $\alpha(x)$ being $n$ bisimulation invariant as well. Also, $\alpha(x)$ is equivalent to some IQML formula if there is some formula $\varphi \in \mathrm{IQML}$ such that for all $\mathcal{M}$ we have $\mathfrak{M}$, $[x \mapsto w] \Vdash \alpha(x)$ iff $\mathcal{M}, w \models \varphi$.

Theorem 4. Let $\alpha(x) \in 2$ Sor.FO with one free variable $x \in \mathcal{V}_{X}$. Then $\alpha(x)$ is bisimulation invariant iff $\alpha(x)$ is equivalent to some IQML formula.

Note that $\Leftarrow$ follows from Theorem 2 . To prove $(\Rightarrow)$ it suffices to show that if $\alpha(x)$ is bisimulation invariant then, for some $n$ it is $n$-bisimulation invariant, since we have already shown in the last section that $n$-bisimulation classes are defined by IQML formulas.

Towards proving this, we introduce a notion of locality for 2Sor.FO formulas. For any tree model $\mathcal{M}$ and let $\mathfrak{M} \mid n$ be the corresponding 2 Sor.FO model of $\mathcal{M}$ restricted to $n$ depth.

Definition 12. We say that a formula $\alpha(x)$ is n-local if for any tree model $(\mathcal{M}, w), \mathfrak{M} \Vdash \alpha(w)$ iff $\mathfrak{M} \mid n \Vdash \alpha(w)$.

Lemma 5. For any $\alpha(x) \in 2$ Sor.FO formula which is bisimulation invariant with $x \in \mathcal{V}_{X}$ then $\alpha(x)$ is n-local for $n=2^{q}$ where $q=q_{x}+q_{\tau}$ where $q_{x}$ is the quantifier rank of $\mathcal{V}_{X}$ sort in $\alpha(x)$ and $q_{\tau}$ is the quantifier rank of $\mathcal{V}_{\tau}$ in $\alpha(x)$. 
Assuming this lemma, consider a 2Sor.FO formula $\alpha(x)$ which is bisimulation invariant. It is $n$-local for a syntactically determined $n$. We now claim that $\alpha(x)$ is $n$-bisimulation invariant. To prove this, consider $\mathcal{M}_{1}, w_{1} \unlhd_{n} \mathcal{M}_{2}, w_{2}$. We need to show that $\mathfrak{M}_{1},\left[x \mapsto w_{1}\right] \Vdash \alpha(x)$ iff $\mathfrak{M}_{2},\left[x \mapsto w_{2}\right] \Vdash \alpha(x)$.

Suppose that $\mathfrak{M}_{1},\left[x \mapsto w_{1}\right] \Vdash \alpha(x)$. By locality, $\mathfrak{M}_{1} \mid n,\left[x \mapsto w_{1}\right] \Vdash \alpha(x)$. Now observe that $\mathcal{M}_{1}\left|n, w_{1} \leftrightarrows \mathcal{M}_{2}\right| n, w_{2}$. By bisimulation invariance of $\alpha(x)$, $\mathfrak{M}_{2} \mid n,\left[x \mapsto w_{2}\right] \Vdash \alpha(x)$. But then again by locality, $\mathfrak{M}_{2},\left[x \mapsto w_{2}\right] \Vdash \alpha(x)$, and we are done.

Thus it only remains to prove the locality lemma. For this, it is convenient to consider the Ehrenfeucht-Fraisse (EF) game for 2Sor.FO. In this game we have two types of pebbles, one for $\mathcal{W}$ and the other for $\mathbb{I}$.

The game is played between two players Spoiler(Sp) and Duplicator(Dup) on two 2Sor.FO structures. A configuration of the game is given by $\left[(\mathfrak{M}, \bar{s}) ;\left(\mathfrak{M}^{\prime}, \bar{t}\right)\right]$ where $\bar{s} \in(\mathcal{W} \cup \mathbb{I})^{*}$ is a finite string $(\mathcal{W} \cup \mathbb{I})$ and similarly $\bar{t} \in\left(\mathcal{W}^{\prime} \cup \mathbb{I}^{\prime}\right)^{*}$.

Suppose the current configuration is $\left[(\mathfrak{M}, \bar{s}) ;\left(\mathfrak{M}^{\prime}, \bar{t}\right)\right]$. In a $\mathcal{W}$ round, Sp places a $\mathcal{W}$ pebble on some $\mathcal{W}$ sort in one of the structures and Dup responds by placing a $\mathcal{W}$ pebble on a $\mathcal{W}$ sort in the other structure. In a I round, similarly Sp picks one structure and places an I pebble on some I sort and Dup responds by placing an $\mathbb{I}$ pebble on some $\mathbb{I}$ sort in the other structure. In both cases, the new configuration is updated to $\left[(\mathfrak{M}, \bar{s} s) ;\left(\mathfrak{M}^{\prime}, \bar{t} t\right)\right]$ where $s$ and $t$ are the new elements(either $\mathcal{W}$ or $\mathbb{I}$ sort) picked in the corresponding structures.

A $\left(q_{x}, q_{\tau}\right)$ round game is one where $q_{x}$ many pebbles of type $\mathcal{W}$ are used and $q_{\tau}$ many pebbles of type $\mathbb{I}$ is used. Player Dup wins after $\left(q_{x}, q_{\tau}\right)$ if after $\left(q_{x}, q_{\tau}\right)$ rounds, if in $\left[(\mathfrak{M}, \bar{s}) ;\left(\mathcal{M}^{\prime}, \bar{t}\right)\right]$ the mapping $f\left(s_{i}\right)=t_{i}$ forms a partial isomorphism over $\mathfrak{M}$ and $\mathfrak{M}^{\prime}$. Otherwise $\mathbf{S p}$ wins.

It can be easily shown that Dup has a winning strategy in the $\left(q_{x}, q_{\tau}\right)$ round game over two structures iff they agree on all formulas with quantifier rank of $\mathcal{V}_{X}$ sort $\leq q_{x}$ and quantifier rank of $\mathcal{V}_{\tau}$ sort $\leq q_{\tau}$.

Let $\mathcal{M}, w$ be any tree structure. To prove lemma 5 , we need to prove that $\mathfrak{M}, w \models \alpha(x)$ iff $\mathfrak{M} \mid n \models \alpha(x)$.

Let $q=q_{x}+q_{\tau}$ and $\mathfrak{N}$ be $q$ disjoint copies of $\mathfrak{M}$ and $\mathfrak{M} \mid n$. Note that inclusion relation $G$ over $\mathfrak{M}$ and $\mathfrak{M} \mid n$ forms a bisimulation. Also note that $G$ continues to be a bisimulation over the disjoint union of $\mathfrak{N} \uplus \mathfrak{M}, w$ and $\mathfrak{N} \uplus \mathfrak{M} \mid n, w$. Moreover, notice that $(\mathfrak{M}, w)$ is bisimilar to $(\mathfrak{N} \uplus \mathfrak{M}, w)$ and further $(\mathfrak{M} \mid n, w)$ is bisimilar to $(\mathfrak{N} \uplus \mathfrak{M} \mid n, w)$.

Now since $\alpha(x)$ is bisimulation invariant, it is enough to show that Dup has a winning strategy in the game starting from $[(\mathfrak{N} \uplus \mathfrak{M}, w),(\mathfrak{N} \uplus \mathfrak{M} \mid n, w)]$.

The winning strategy for Dup is to ensure that at every round $m<\left(q_{x}+q_{\tau}\right)$ the critical distance $d_{m}=2^{q-m}$ is respected:

If $\mathbf{S p}$ places $\mathcal{W}$ pebble on a $\mathcal{W}$ sort which is within $d_{m}$ of an already pebbled $\mathcal{W}$ pebble, Dup plays according to a local isomorphism in the $d_{m^{-}}$neighbourhoods of previously pebbled elements (exists since $n=2^{q}$ and $m<q$ ); if $\mathbf{S p}$ places a $\mathcal{W}$ pebble somewhere beyond $2^{q-m}$ distance from all $\mathcal{W}$ pebbles previously used, then, Dup responds in a fresh isomorphic copy of type $\mathfrak{M}$ or 
$\mathfrak{M} \mid n$ correspondingly (again, it is guaranteed to exist since previously at most $m-1(<q)$ would have been used).

If $\mathbf{S p}$ decides to use an I pebble and places it on some I sort $i$ in one structure, then Dup responds by placing an II pebble on $i$ in the mirror copy in the other structure, where by mirror copy we mean: for $\mathfrak{M}$ or $\mathfrak{M} \mid n$ in $\mathfrak{N}$ then the mirror copy in the other structure is itself and the original $\mathfrak{M}$ and $\mathfrak{M} \mid n$ are mirror copies of each other.

\section{Satisfiability problem}

The satisfiability problem for IQML can be solved by sharpening the completeness proof of the axiom system by showing that every consistent formula is satisfied in a model of bounded size. Indeed, a PSPACE decision procedure can be given along the lines of Grove and Halpern [12. However, we give a tableau procedure for IQML which is instructive, and as we will observe later, neatly generalizes to more expressive logics.

Given a formula $\varphi$, we set $I=\left\{c_{\alpha} \mid\langle\exists\rangle \alpha \in S F(\varphi)\right\} \cup\left\{d_{\beta} \mid[\exists] \beta \in S F(\varphi)\right\}$ where $S F(\varphi)$, the set of subfomulas of $\varphi$ is defined in the standard way. This forms the index set where $c_{\alpha}$ and $d_{\beta}$ act as witnesses for the corresponding formulas.

We construct a tableau tree structure $T=(W, V, E, \lambda)$ where $W$ is a finite set, $(V, E)$ is a rooted tree and $\lambda: V \mapsto L$ is a labelling map. Each element in $L$ is of the form $\left(w: \Gamma, i_{\chi}\right)$, where $w \in W, \Gamma$ is a finite set of formulas and $i_{\chi} \in I$. The intended meaning of the label is that the node constitutes a world $w$ that satisfies the formulas in $\Gamma$ and $i_{\chi}$ is the incoming label edge of $w$.

The tableau rules for IQML are inspired from the tableau procedure for the bundled fragment of first order modal logic introduced in [19]. The $(\wedge)$ and $(\vee)$ tableau rules are standard. For the modalities, the intuition for the corresponding tableau rule is the following: Suppose that we are in an intermediate step of tableau construction when we have formulas $\{\langle\exists\rangle \alpha,[\exists] \beta,\langle\forall\rangle \varphi,[\forall] \psi\}$ to be satisfied at a node $w$. For this, first we need to add a new $c_{\alpha}$ successor node $w v_{\alpha}$ where $\alpha$ holds; this new node inherits not only $\alpha$ but also $\psi$. Also, we need a $d_{\beta}$ successor which inherits $\beta, \varphi$ and $\psi$. Finally for each $e_{\gamma} \in I$ we need a $\varphi$-successor which also inherits $\psi$.

The (BR) tableau rule extends this idea when there are multiple occurrences of each kind of formulas above. In general if the set of formulas considered at node $w:(A, B, C, D)$ where $A=\left\{\langle\exists\rangle \alpha_{1} . .\langle\exists\rangle \alpha_{n_{1}}\right\} ; B=\left\{[\exists] \beta_{1} . .[\exists] \beta_{n_{2}}\right\} ; C=$ $\left\{\langle\forall\rangle \varphi_{1} . .\langle\forall\rangle \varphi_{m_{1}}\right\}$ and $D=\left\{[\forall] \psi_{1} . .[\forall] \psi_{m_{2}}\right\}$. Let $D^{\prime}=\{\psi \mid[\forall] \psi \in D\}$. The BR rule is given as follows:

$$
\begin{gathered}
\frac{w:(A, B, C, D)}{\left\{\left\langle w v_{\alpha_{i}}:\left(\alpha_{i}, D^{\prime}\right), c_{\alpha_{i}}\right\rangle \mid i \leq n_{1}\right\} \cup}(\mathrm{BR}) \\
\left\{\left\langle w v_{\beta_{j}}^{k}:\left(\beta_{j}, \varphi_{k}, D^{\prime}\right), d_{\beta_{j}}\right\rangle \mid k \leq m_{1}, j \leq n_{2}\right\} \cup \\
\left\{\left\langle w v_{e_{\chi}}^{k}:\left(\varphi_{k}, D\right), e_{\chi}\right\rangle \mid l \leq m_{1}, \chi \notin(A \cup B)\right\}
\end{gathered}
$$


From an 'open tableau' we can construct a model for $\varphi$, along the lines of [19]. Conversely it can be proved that every satisfiable formula has an open tableau.

This tableau construction can be extended to the 'bundled fragment' of full TML where we have predicates of arbitrary arity and the quantifiers and modalities occur (only) in the form $\forall x \square_{x} \varphi$ and $\exists x \square \varphi$ (and their duals). The proof follows the lines of $[19$.

\section{Discussion}

We have studied the variable-free fragment of PTML, with implicit modal quantification. We could also consider more forms of implicit quantification such as $\square \forall$ and $\diamond \forall$ modalities, though there is no obvious semantics to them. These logics are the obvious variable free versions of monadic 'bundled' fragments of TML. One could consider a similar exercise for 'bundled' fragments of first order modal logic (FOML). As [19] shows, this is a decidable logic for increasing domain semantics.

Our study suggests that there are other forms of implicitly quantified modal logics. For instance, is there an implicit hybrid version of the logic studied by Wang and Seligman [23]?

A natural question is the delimitation of expressiveness of these logics: which are the properties of models expressed only by $\exists \square$ or only by $\forall \square$ modalities ? How does nesting of these modalities increase expressive power ? We believe that the model theory of implicit modal quantification may offer interesting possibilities for abstract specifications of some infinite-state systems. However, for such study, we will need to consider transitive closures of accessibility relations, and this seems to be quite challenging.

Recent developments in tools for model checking and other decision procedures for fragments of FOML offer a promising direction to develop similar practical frameworks for IQML and other decidable fragments of term-modal logics. Such tools can be of help in the synthesis and verification of some classes of systems with unboundedly many agents.

Acknowledgement. We thank Yanjing Wang for his insightful and extensive discussions on the theme of this paper.

\section{References}

1. Alur, R., Henzinger, T.A., Kupferman, O.: Alternating-time temporal logic. J. ACM 49(5), 672-713 (2002). https://doi.org/10.1145/585265.585270

2. van Benthem, J., et al.: Frame correspondences in modal predicate logic. Proofs, categories and computations: Essays in honor of Grigori Mints pp. 1-14 (2010)

3. Blackburn, P.: Nominal tense logic. Notre Dame Journal of Formal Logic 34(1), 56-83 (1993). https://doi.org/10.1305/ndjfl/1093634564 http://dx.doi.org/10.1305/ndjfl/1093634564

4. Blackburn, P., de Rijke, M., Venema, Y.: Modal Logic (Cambridge Tracts in Theoretical Computer Science). Cambridge University Press (2001) 
5. Clarke, E.M., Emerson, E.A., Sistla, A.P.: Automatic verification of finite-state concurrent systems using temporal logic specifications. ACM Transactions on Programming Languages and Systems (TOPLAS) 8(2), 244-263 (1986)

6. van Ditmarsch, H., van der Hoek, W., Kooi, B.: Dynamic Epistemic Logic: 337 (Synthese Library). Springer Netherlands (2007)

7. Fagin, R., Halpern, J.Y., Moses, Y., Vardi, M.: Reasoning About Knowledge. A Bradford Book (2004)

8. Fitting, M., Mendelsohn, R.L.: First-Order Modal Logic (Synthese Library). Springer (1999)

9. Fitting, M., Thalmann, L., Voronkov, A.: Term-modal logics. Studia Logica 69(1), 133-169 (2001). https://doi.org/10.1023/A:1013842612702

10. Gargov, G., Goranko, V.: Modal logic with names. J. Philosophical Logic 22(6), 607-636 (1993). https://doi.org/10.1007/BF01054038 http://dx.doi.org/10.1007/BF01054038

11. Grove, A.J.: Naming and identity in epistemic logic part ii: a first-order logic for naming. Artificial Intelligence 74(2), 311-350 (1995)

12. Grove, A.J., Halpern, J.Y.: Naming and identity in epistemic logics part i: the propositional case. Journal of Logic and Computation 3(4), 345-378 (1993)

13. van der Hoek, W., Pauly, M.: 20 modal logic for games and information. In: Studies in Logic and Practical Reasoning, vol. 3, pp. 1077-1148. Elsevier (2007)

14. Hughes, M., Cresswell, G.: A New Introduction to Modal Logic. Routledge. 1996. Routledge (1996)

15. Khan, M.A., Banerjee, M., Rieke, R.: An update logic for information systems. Int. J. Approx. Reasoning 55(1), 436-456 (2014). https://doi.org/10.1016/j.ijar.2013.07.007

16. Kooi, B.: Dynamic term-modal logic. In: A Meeting of the Minds. pp. 173-186 (2007)

17. Orlandelli, E., Corsi, G.: Decidable term-modal logics. In: 15th European Conference on Multi-Agent Systems (2017)

18. Padmanabha, A., Ramanujam, R.: The monodic fragment of propositional term modal logic. Studia Logica pp. 1-25 (2018)

19. Padmanabha, A., Ramanujam, R., Wang, Y.: Bundled fragments of first-order modal logic: (un)decidability. In: 38th IARCS Annual Conference on Foundations of Software Technology and Theoretical Computer Science, FSTTCS 2018, December 11-13, 2018, Ahmedabad, India. pp. 43:1-43:20 (2018). https://doi.org/10.4230/LIPIcs.FSTTCS.2018.43

20. Passy, S., Tinchev, T.: Quantifiers in combinatory PDL: completeness, definability, incompleteness. In: Fundamentals of Computation Theory, FCT '85, Cottbus, GDR, September 9-13, 1985. pp. 512-519 (1985). https://doi.org/10.1007/BFb0028835, http://dx.doi.org/10.1007/BFb0028835

21. Shtakser, G.: Propositional epistemic logics with quantification over agents of knowledge. Studia Logica 106(2), 311-344 (2018)

22. Wang, Y.: A new modal framework for epistemic logic. In: Proceedings Sixteenth Conference on Theoretical Aspects of Rationality and Knowledge, TARK 2017, Liverpool, UK, 24-26 July 2017. pp. 515-534 (2017). https://doi.org/10.4204/EPTCS.251.38

23. Wang, Y., Seligman, J.: When names are not commonly known: Epistemic logic with assignments. In: Advances in Modal Logic Vol. 12 (2018): 611-628, College Publications 